\title{
Climatic impacts of stochastic fluctuations in air-sea fluxes
}

\author{
Paul D. Williams ${ }^{1}$ \\ Received 26 March 2012; revised 20 April 2012; accepted 22 April 2012; published 23 May 2012.
}

[1] Air-sea fluxes vary partly on scales that are too small or fast to be resolved explicitly by global climate models. This paper proposes a nonlinear physical mechanism by which stochastic fluctuations in the air-sea buoyancy flux may modify the mean climate. The paper then demonstrates the mechanism in climate simulations with a comprehensive coupled general circulation model. Significant changes are detected in the time-mean oceanic mixed-layer depth, seasurface temperature, atmospheric Hadley circulation, and net upward water flux at the sea surface. Also, El Niño Southern Oscillation (ENSO) variability is significantly increased. The findings demonstrate that noise-induced drift and noiseenhanced variability, which are familiar concepts from simple climate models, continue to apply in comprehensive climate models with millions of degrees of freedom. The findings also suggest that the lack of representation of sub-grid variability in air-sea fluxes may contribute to some of the biases exhibited by contemporary climate models. Citation: Williams, P. D. (2012), Climatic impacts of stochastic fluctuations in air-sea fluxes, Geophys. Res. Lett., 39, L10705, doi:10.1029/2012GL051813.

\section{Introduction}

[2] The atmosphere and ocean are coupled by fluxes of energy, momentum, and matter across the air-sea interface [e.g., Peixóto and Oort, 1984]. Air-sea fluxes are observed to vary on a vast range of scales in both space and time [e.g., Sun et al., 1996]. Part of the variability takes place on scales that are too small or fast to be resolved explicitly by the numerical grids and time steps of contemporary global climate models. For example, sub-grid fluctuations in precipitation and short-wave solar radiation may occur because of convective clouds, and sub-grid fluctuations in evaporation, latent heat, and momentum may occur because of turbulence in the surface wind stress. These fluctuations may play an important role in climate, despite their high frequencies and short spatial scales. For example, increasing the temporal resolution of the air-sea fluxes in a coupled atmosphereocean model, in order to resolve more of the high-frequency exchanges, affects various aspects of the simulated climate [Bernie et al., 2005].

[3] In climate models, how best to parameterize the subgrid air-sea fluxes remains unclear. Stochastic closure schemes generally have a firmer theoretical basis than their deterministic counterparts and appear to hold more promise [e.g., Palmer, 2001; Williams et al., 2004; Williams, 2005;

\footnotetext{
${ }^{1}$ Department of Meteorology, University of Reading, Reading, UK.

Corresponding author: P. D. Williams, Department of Meteorology, University of Reading, Earley Gate, Reading RG6 6BB, UK. (p.d.williams@reading.ac.uk)

Copyright 2012 by the American Geophysical Union. 0094-8276/12/2012GL051813
}

Palmer and Williams, 2008; Stolle et al., 2009; Chekroun et al., 2011]. In atmosphere models, stochastic perturbations are applied routinely in many operational numerical weather prediction centers [e.g., Buizza et al., 1999, 2005]. In ocean models, stochastic air-sea fluxes derived from an empirical model of atmospheric dynamics deepen the mixed layer [Balan Sarojini and von Storch, 2009] and stochastic forcing prolongs the duration of deep convection compared to deterministic forcing [Kuhlbrodt and Monahan, 2003]. Stochastic perturbations are absent from most coupled atmosphere-ocean general circulation models used for climate simulation and prediction, however.

[4] In simple climate models with only a few degrees of freedom, the effects of external stochastic noise are well understood, through the application of theoretical tools including the Fokker-Planck equation. The effects may be categorized into three broad classes. First, the mean climate may be modified, through a noise-induced drift [e.g., Sardeshmukh et al., 2003]. Second, the climate variability may be enhanced, just as the Brownian motion of a pollen grain is made more variable by random buffeting from the molecules of the fluid in which it is suspended [e.g., Wilks, 2008]. Finally, the climate may undergo noise-activated regime transitions between different stable states [e.g., Birner and Williams, 2008]. However, although simple climate models are useful for gaining a mechanistic understanding of a small number of processes operating in isolation, they are unsuitable for quantitative climate simulation and prediction, for which comprehensive climate models must be used instead.

[5] In comprehensive climate models with millions of degrees of freedom, the effects of external stochastic noise are not as well understood, because analytic progress using the Fokker-Planck equation and other theoretical tools is impossible. Correspondingly, the extent to which the above noise-induced concepts are relevant in comprehensive models is still an area of active research. As evidence of noise-induced drift in a comprehensive model, Palmer [2001] reports that the cold tropical sea-surface temperature bias in the coupled model of the European Centre for Medium-Range Weather Forecasts (ECMWF) is reduced by around $0.2^{\circ} \mathrm{C}$ when an atmospheric stochastic physics parameterization is implemented. As evidence of noiseactivated regime transitions in a comprehensive model, Wang et al. [1999] find that the timing of a collapse of the ocean's thermohaline circulation is affected by stochastic fluctuations applied to the wind stress field. Much remains unknown about the extent to which stochastic fluctuations may modify climate simulations, however.

[6] This paper proposes a particular physical mechanism by which stochastic fluctuations in the air-sea fluxes may modify the time-mean climate, even though the time-mean fluctuation is zero (by definition). The paper then demonstrates the proposed mechanism in simulations with a 
comprehensive coupled general circulation model. The paper finds evidence of noise-induced drift and noise-enhanced variability, even in a model of this complexity. The layout of the paper is as follows. The conjectured mechanism is described in section 2. The experimental design for the numerical simulations is described in section 3 . The impacts of the stochastic fluctuations, on both the time-mean climate and the temporal variability, are described in section 4 . The paper concludes with a summary and discussion in section 5 .

\section{Conjectured Mechanism}

[7] This section proposes a nonlinear physical mechanism by which the noise-induced drift effect may operate when the air-sea fluxes contain a stochastic component. The conjectured mechanism permits stochastic fluctuations in the air-sea buoyancy fluxes to modify the time-mean mixedlayer depth. The mixed-layer depth is generally determined by the combined influence of surface buoyancy fluxes and turbulent winds, but only the surface buoyancy fluxes are invoked here, because the response to stochastic winds has been studied elsewhere [Wang et al., 1999]. Note that the importance of surface buoyancy fluxes is not confined to the polar regions, where surface cooling triggers deep convective events, but extends to all latitudes.

[8] The proposed mechanism relies on a fundamental asymmetry in the physics of the ocean mixed layer, as follows. In a statically stable water column in the mixed layer, dense anomalies at the surface (created by evaporation or cooling) can destabilize the water column, initiate convection and vertical mixing, and deepen the mixed layer. However, in contrast, buoyant anomalies at the surface (created by precipitation or heating) simply further stabilize the water column and cannot shoal the mixed layer. Therefore, the ocean mixed-layer depth responds asymmetrically to positive and negative surface buoyancy fluctuations. In short, positive fluctuations cannot undo the vertical mixing caused by negative fluctuations of equal magnitude. The mechanism has much in common with the mixed-layer demon of Stommel [1979], who proposed that "there is some process at work that selects only late winter water for actual net downward pumping", "a process much like that performed by Maxwell's Demon". Stommel's hypothesis is generally supported by ocean model studies [e.g., Williams et al., 1995].

[9] Note that the conjectured asymmetry is important only near the threshold for static instability. However, the mixed layer is always near this threshold, because it is only weakly stratified. For example, the observed diurnal cycle of mixedlayer depth, which consists of nightly deepening caused by surface heat loss to the atmosphere [e.g., Bernie et al., 2005], would not occur if the threshold were not near. Therefore, the threshold for static instability may easily be reached by the surface buoyancy perturbations being represented stochastically in the present study.

[10] Stochastic fluctuations in the air-sea buoyancy flux are expected to have various impacts, in sequence, on the time-mean climate. First, because of the above mechanism, the mixed layer is expected to deepen systematically, consistent with Balan Sarojini and von Storch [2009]. Then, contact with deeper water is expected to cool the mixed layer, especially on the thermal equator where the mixed layer is shallowest. Then, the atmospheric Hadley circulation in each hemisphere is expected to weaken, because warm equatorial surface water provides the thermal energy for these circulations [e.g., Bjerknes, 1966]. Finally, the weakened Hadley circulations are expected to decrease precipitation in the inter-tropical convergence zone (ITCZ), because of decreased horizontal convergence and decreased upward flow, and to increase precipitation in the subtropical high pressure regions at around $30^{\circ} \mathrm{N}$ and $30^{\circ} \mathrm{S}$, because of decreased downward flow. The remainder of this paper demonstrates these hypothesized impacts in simulations with a coupled general circulation model.

\section{Experimental Design}

[11] The numerical experiments are performed using the SINTEX-G coupled atmosphere-ocean model [Gualdi et al., 2008]. The atmosphere component, version 4.6 of ECHAM [Roeckner et al., 1996], integrates the primitive equations on a T30L19 grid with a time step of $30 \mathrm{~min}$. The ocean component, the global configuration of version 8.2 of OPA [Madec et al., 1998], integrates the hydrostatic primitive equations with a time step of $90 \mathrm{~min}$, a longitudinal resolution of $2.0^{\circ}$, a latitudinal resolution ranging from $0.5^{\circ}$ at the equator to $2.0^{\circ}$ at the poles, and a vertical resolution ranging from $10 \mathrm{~m}$ at the sea surface to $500 \mathrm{~m}$ at the sea floor. In OPA, air-sea fluxes directly affect the top ocean model layer, and the mixed layer emerges as the result of exchanges between the top few model layers. Explicit vertical mixing associated with static instabilities and convection is absent because of the hydrostatic approximation, but it is parameterized by a non-penetrative convective-adjustment scheme. A sea-ice model is included, and the ocean and sea ice are coupled to the atmosphere every $180 \mathrm{~min}$, using version 2.4 of the OASIS coupling software [Valcke et al., 2000]. No flux adjustment is applied, except in the Mediterranean Sea and the Red Sea, which are not well resolved. The global water cycle is closed using the river-catchment scheme developed by Williams et al. [2010].

[12] Three SINTEX-G simulations are run, each 100 years long and initiated from the Levitus [1982] ocean observations. The three simulations consist of two experimental runs, in which the water and heat components of the buoyancy flux are perturbed separately, and a control run. In the first perturbation experiment, hereafter labeled WAT, the deterministically calculated net fresh water flux (i.e., liquid precipitation minus evaporation) across the air-sea interface is modified stochastically before being passed to the ocean. In the second perturbation experiment, hereafter labeled HEA, the deterministically calculated net heat flux (i.e., the sum of short-wave radiation, long-wave radiation, sensible heat, and latent heat) across the air-sea interface is modified stochastically before being passed to the ocean. In the control experiment, hereafter labeled CTL, the net fresh water flux and the net heat flux are unmodified.

[13] The stochastic modifications are achieved through multiplication by spatially uncorrelated white noise drawn, at each ocean grid box and at each coupling time step, from a uniform distribution between 0.5 and 1.5. Note that multiplicative noise is used instead of additive noise, following previous work [e.g., Buizza et al., 1999]. If additive noise were used, then the noise-induced drift effect would be absent, and diffusion of probability by noise would be the only mechanism that could change the statistics of the 


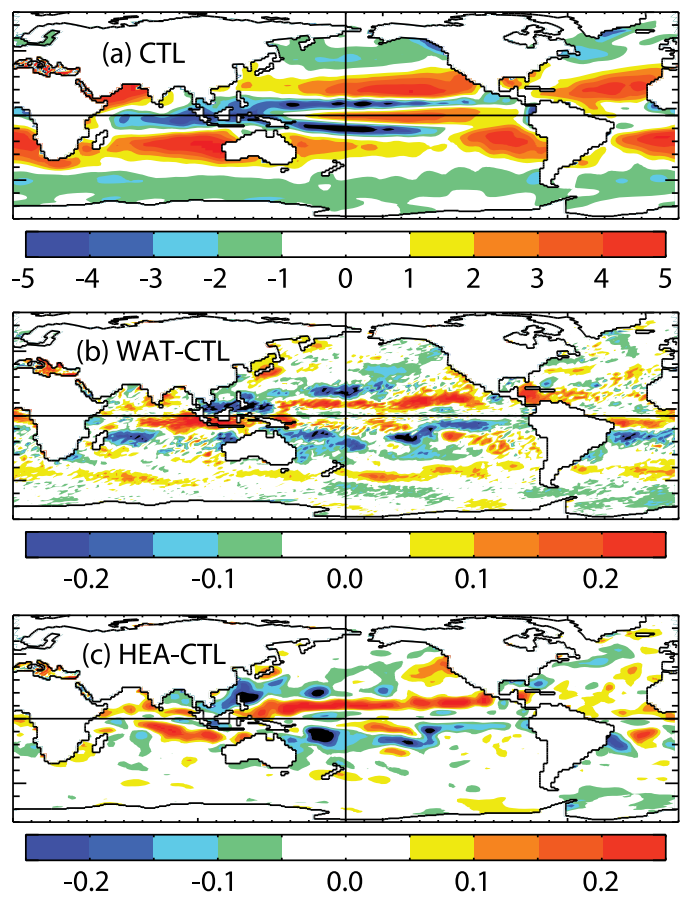

Figure 1. Maps of the century-mean net upward water flux $(\mathrm{mm} /$ day) at the sea surface in (a) CTL, (b) WAT - CTL, and (c) HEA-CTL. Note that Figure 1a shows the control simulation and Figures $1 \mathrm{~b}$ and $1 \mathrm{c}$ show anomalies in the experimental simulations with respect to the control. Positive values indicate (anomalous) net evaporation and negative values indicate (anomalous) net precipitation.

system. The importance of multiplicative noise in stochastic climate models has been stressed by Majda et al. [2001].

[14] In WAT, the net surface fresh water flux (which includes a contribution from convective rain) is being perturbed, but the convection parameterization in the atmosphere model is not being perturbed consistently. Similarly, in HEA, the net surface heat flux (which includes a longwave contribution) is being perturbed, but the cloud scheme in the model is not being perturbed consistently. Therefore, during single time steps and at individual grid points, water and energy are effectively being created and destroyed at the sea surface. This violation of local conservation is normal in sensitivity experiments designed to study a specific process in isolation [e.g., Williams et al., 2007]. Importantly, the law of large numbers ensures that the average perturbation over many time steps or grid points is essentially zero, guaranteeing the long-term, global conservation of water and energy.

\section{Experimental Results}

[15] This section documents the impacts of the stochastic air-sea fluxes on various aspects of the century-mean climate. The oceanic mixed-layer depth, sea-surface temperature, atmospheric Hadley circulation, and net upward water flux at the sea surface will each be considered, in turn. Each of the century-mean differences reported in this section is statistically significant at the $95 \%$ level at least, according to two-tailed $t$ tests.

[16] The century-mean mixed layer is generally deeper in each stochastic experiment than in the control run, consistent with the mechanism conjectured in section 2. The relative amount by which the mixed layer deepens is largest in the tropics, especially near the equator where the control mixed layer is shallowest. For example, along the thermal equator in the Pacific Ocean (averaged over $5-10^{\circ} \mathrm{N}$ ), the centurymean mixed layer is $33.4 \mathrm{~m}$ deep in CTL, but it deepens by $0.8 \mathrm{~m}(\sim 2 \%)$ in WAT and by $1.0 \mathrm{~m}(\sim 3 \%)$ in HEA. The bottom of the mixed layer is computed here as the depth at which the density exceeds the local surface density by $0.01 \mathrm{~kg} \mathrm{~m}^{-3}$. In the annual-mean data, the mixed-layer depth anomalies are present in WAT and HEA from the first year, as expected for this mechanism.

[17] The century-mean tropical ocean surface generally cools in WAT and HEA, which is consistent with the mixedlayer depth changes, because the water being mixed up from beneath the mixed layer is cooler. The maximum surface cooling in WAT and HEA is a few tenths of a degree Celsius along the thermal equator. This cooling is small, but the atmospheric circulation is very sensitive to sea-surface temperature on the equator. As a consequence, the centurymean Hadley cells in each hemisphere weaken in WAT and HEA. For example, averaged over the southward surface branch of the northern meridional overturning cell (10 $30^{\circ} \mathrm{N}$ ) in the Pacific Ocean, the century-mean meridional surface wind stress exerted by the atmosphere on the ocean is $28.8 \times 10^{-3} \mathrm{~N} \mathrm{~m}^{-2}$ southward in CTL, but it is $1.1 \times 10^{-3} \mathrm{~N} \mathrm{~m}^{-2}(\sim 4 \%)$ weaker in WAT and $1.0 \times$ $10^{-3} \mathrm{~N} \mathrm{~m}^{-2}(\sim 3 \%)$ weaker in HEA. Similarly, averaged over the northward surface branch of the southern meridional overturning cell $\left(10-30^{\circ} \mathrm{S}\right)$ in the Pacific Ocean, the meridional surface wind stress exerted by the atmosphere on the ocean is $14.8 \times 10^{-3} \mathrm{~N} \mathrm{~m}^{-2}$ northward in CTL, but it is $0.9 \times 10^{-3} \mathrm{~N} \mathrm{~m}^{-2}(\sim 6 \%)$ weaker in WAT and $0.8 \times 10^{-3} \mathrm{~N} \mathrm{~m}^{-2}(\sim 5 \%)$ weaker in HEA.

[18] Maps of the century-mean net upward water flux (i.e., evaporation minus precipitation) at the sea surface are shown in Figure 1, and plots of the zonal mean are shown in Figure 2. The equatorial upward branches of the Hadley cells tend to promote precipitation, and the subtropical downward branches tend to inhibit precipitation. Therefore, when the Hadley cells are weakened in WAT and HEA, the equatorial precipitation is decreased and the subtropical precipitation is increased, as seen in the figures. For example, along the inter-tropical convergence zone in the Pacific Ocean (averaged over $7-12^{\circ} \mathrm{N}$ ), the net precipitation is $3.3 \mathrm{~mm}$ day $^{-1}$ in CTL, but it is reduced by $0.2 \mathrm{~mm}$ day $^{-1}$ $(\sim 6 \%)$ in WAT and HEA. This is a rectification effect, and the anti-correlation of the net upward water flux between the control and each experiment is clear by visual inspection of the figures. For example, the linear Pearson pattern correlation coefficient computed between CTL and WAT-CTL (i.e., between Figures 1a and $1 \mathrm{~b}$ ) is -0.29 in the Pacific Ocean; the $95 \%$ confidence interval is -0.25 to -0.33 . Also, the correlation coefficient computed between the zonal means in CTL and WAT-CTL (i.e., between Figures 2a and $2 b$ ) is -0.66 ; the $95 \%$ confidence interval is -0.51 to -0.77 . These anti-correlations occur because the Hadley circulation's promotion of precipitation on the equator and inhibition of precipitation in the subtropics are reduced in WAT and HEA compared to CTL.

[19] Note that, despite the very different spatial patterns of the stochastic forcing imposed in WAT and HEA, the response of the century-mean climate is similar. For example, 

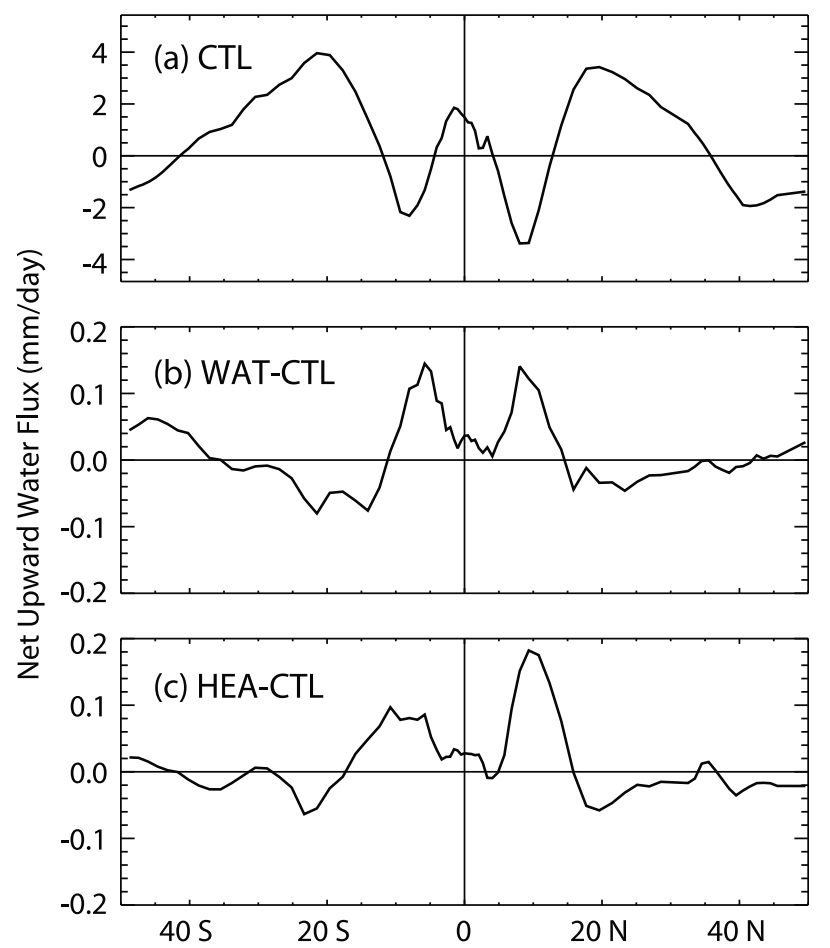

Figure 2. Plots of the zonal-mean century-mean net upward water flux (mm/day) at the sea surface in (a) CTL, (b) WAT-CTL, and (c) HEA-CTL. Note that Figure 2a shows the control simulation and Figures $2 \mathrm{~b}$ and $2 \mathrm{c}$ show anomalies in the experimental simulations with respect to the control. Positive values indicate (anomalous) net evaporation and negative values indicate (anomalous) net precipitation.

in the net upward water flux, the global pattern correlation coefficient between WAT-CTL and HEA-CTL (i.e., between Figures $1 \mathrm{~b}$ and $1 \mathrm{c}$ ) is +0.53 ; the $95 \%$ confidence interval is +0.52 to +0.54 . Also, the correlation coefficient computed between the zonal means in WAT-CTL and HEA-CTL (i.e., between Figures $2 \mathrm{~b}$ and 2c) is +0.68 ; the $95 \%$ confidence interval is +0.54 to +0.79 . This similarity occurs because stochastic perturbations to the air-sea buoyancy flux, with any spatial pattern, will always systematically deepen the mixed layer according to the mechanism conjectured in section 2 .

[20] Externally imposed noise is generally expected to increase the variability of dynamical systems, as well as to change the mean. In the present experiments, the largest increases in the variability are found in the sea-surface temperature field at El Niño Southern Oscillation (ENSO) frequencies. This is perhaps unsurprising, because ENSO depends crucially on interactions between the atmosphere and ocean, which are mediated by the air-sea fluxes being modified here. Figure 3 shows global maps of the sea-surface temperature variability at the center of the ENSO frequency band (i.e., for periods between $3.6 \mathrm{yr}$ and $4.5 \mathrm{yr}$ ). ENSO variability in the eastern equatorial Pacific Ocean is clearly increased by the stochastic forcing in WAT and HEA. Compared to CTRL, the amplitude of the sea-surface temperature cycle in this frequency band and in the Niño-3 region $\left(90-150^{\circ} \mathrm{W}, 5^{\circ} \mathrm{S}-5^{\circ} \mathrm{N}\right)$ increases by $110 \%$ in WAT and $160 \%$ in HEA. The corresponding variance increases are $230 \%$ and $410 \%$, respectively. In the broader ENSO frequency band (i.e., for periods between $2.0 \mathrm{yr}$ and $7.0 \mathrm{yr}$ ), the amplitude of the sea-surface temperature cycle in the Niño-3 region increases by $20 \%$ in WAT and $30 \%$ in HEA. The corresponding variance increases are $40 \%$ and $60 \%$, respectively. These differences are each statistically significant at the $95 \%$ level, according to two-tailed $F$ tests.

\section{Summary and Discussion}

[21] In this study, stochastic fluctuations have been applied to the air-sea buoyancy fluxes in a comprehensive climate model. Unlike related previous work, which has employed an ocean general circulation model coupled only to a simple empirical model of atmospheric dynamics [Balan Sarojini and von Storch, 2009], the present work has employed a full coupled atmosphere-ocean general circulation model. This advance allows the feedbacks in the coupled system to be captured as comprehensively as is permitted by contemporary high-performance computing, and it allows the impacts on the atmospheric circulation to be studied. The stochastic fluctuations were introduced as a crude attempt to capture the variability of rapid, sub-grid structures otherwise missing from the model. Experiments have been performed to test the response of the climate system to the stochastic noise.

[22] In two experiments, the net fresh water flux and the net heat flux were perturbed separately. Significant changes were detected in the century-mean oceanic mixed-layer depth, sea-surface temperature, atmospheric Hadley circulation, and net upward water flux at the sea surface. Significant changes were also detected in the ENSO variability. The century-mean changes are summarized schematically in Figure 4. The above findings constitute evidence that noise-induced drift and noise-enhanced variability, which are familiar concepts from simple models, continue to apply in comprehensive climate models with millions of degrees of

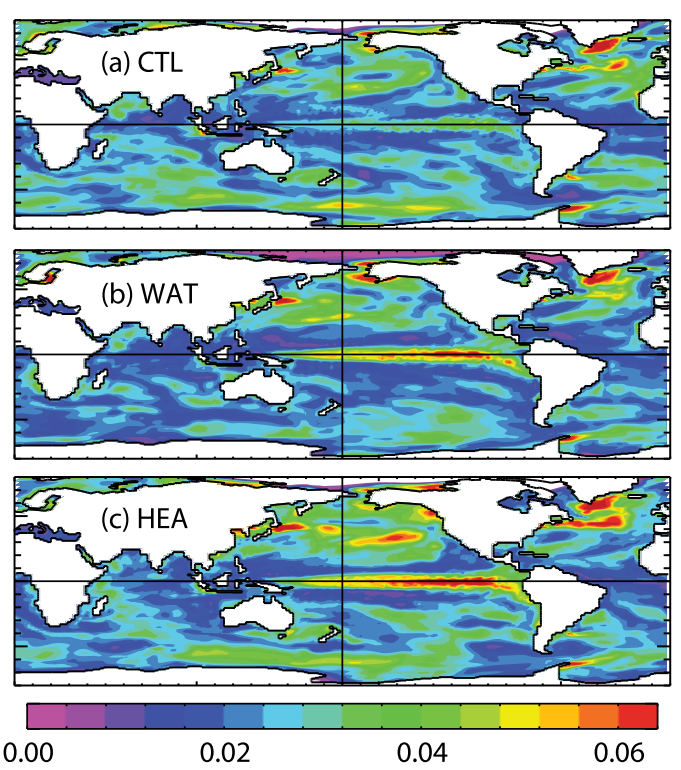

Figure 3. Maps of the variability of sea-surface temperature $\left({ }^{\circ} \mathrm{C}\right.$ ) in (a) CTL, (b) WAT, and (c) HEA. The quantity shown is the magnitude of the Fourier coefficients obtained from 100-year time series of monthly mean sea-surface temperature. The Fourier coefficients have been averaged over periods from $3.6 \mathrm{yr}$ to $4.5 \mathrm{yr}$. 


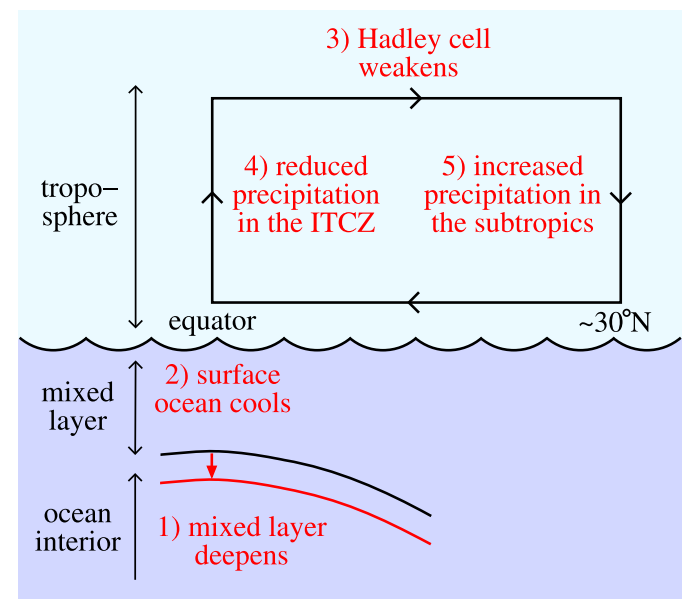

Figure 4. Schematic diagram summarising the time-mean, zonal-mean response of the low-latitude ocean and atmosphere to stochastic air-sea flux perturbations. Only northern latitudes are shown, but the response is symmetric about the (thermal) equator.

freedom. Note that the noise-induced drift and the mixedlayer asymmetry are complementary interpretations of the changes from different view points: the former is the generic mathematical explanation in the context of the Fokker-Plank equation, and the latter is the particular physical mechanism.

[23] The coupled atmosphere-ocean general circulation models used for contemporary climate simulation and prediction exhibit significant biases with respect to the observed climate system. These biases occur in both the mean state and the variability. An implication of the findings of this study is that the lack of representation of sub-grid variability in the airsea fluxes may contribute to some of these biases. For example, compared to the Levitus [1982] ocean observations, in the tropical Pacific ocean the SINTEX-G model exhibits a cold surface bias of up to around $1^{\circ} \mathrm{C}$ in the west and a warm surface bias of up to around $1{ }^{\circ} \mathrm{C}$ in the east. The tropical surface cooling caused by the stochastic perturbations in this study deteriorates the former bias but improves the latter. Therefore, this study adds to the existing evidence [e.g., Bernie et al., 2005] for the importance of the faithful representation of high-frequency air-sea fluxes. This study also adds to the existing evidence [e.g., Palmer and Williams, 2008] for the utility of stochastic approaches to climate modeling.

[24] It is instructive to compare and contrast the stochastic perturbations applied in the present study with those used operationally at the European Centre for Medium-Range Weather Forecasts [Buizza et al., 1999]. In both cases, the noise is multiplicative and is drawn from $U(0.5,1.5)$. In the present study, however, the noise is uncorrelated in space and time. This assumption is appropriate, because this is the first study to examine the climatic impacts of stochastic perturbations to the air-sea buoyancy fluxes in a comprehensive coupled atmosphere-ocean general circulation model. Therefore, the present work is to be regarded as a proof-of-concept study, which could be refined in future work. For example, additive noise could be used instead of multiplicative noise, to determine whether state-dependent stochastic perturbations are essential for the impacts documented herein. Also, red noise could be used instead of white noise, with a correlation time scale and length scale derived from weather statistics or other physical insights. Consistent with expectations from simple models and Brownian motion, it is speculated that the use of correlated noise would increase the magnitude of the impacts on the mean state and variability.

[25] Acknowledgments. The author is funded through a University Research Fellowship from the Royal Society (reference: UF080256). Supercomputer calculations were performed on the NEC SX-8 at the Institut du Développement et des Ressources en Informatique Scientifique (IDRIS/ CNRS) in Paris and the NEC SX-6 at the Deutsches Klimarechenzentrum (DKRZ/MPI-M) in Hamburg. The comments of two anonymous reviewers are gratefully acknowledged.

[26] The Editor thanks two anonymous reviewers for their assistance evaluating this manuscript.

\section{References}

Balan Sarojini, B., and J.-S. von Storch (2009), Effects of fluctuating daily surface fluxes on the time-mean oceanic circulation, Clim. Dyn., 33(1), 1-18, doi:10.1007/s00382-009-0575-y.

Bernie, D. J., S. J. Woolnough, J. M. Slingo, and E. Guilyardi (2005), Modeling diurnal and intraseasonal variability of the ocean mixed layer, J. Clim., 18(8), 1190-1202, doi:10.1175/JCLI3319.1.

Birner, T., and P. D. Williams (2008), Sudden stratospheric warmings as noise-induced transitions, J. Atmos. Sci., 65(10), 3337-3343, doi:10.1175/ 2008JAS2770.1.

Bjerknes, J. (1966), A possible response of the atmospheric Hadley circulation to equatorial anomalies of ocean temperature, Tellus, 18(4), $820-829$.

Buizza, R., M. Miller, and T. N. Palmer (1999), Stochastic representation of model uncertainties in the ECMWF ensemble prediction scheme, $Q$. J. R Meteorol. Soc., 125, 2887-2908.

Buizza, R., P. L. Houtekamer, Z. Toth, G. Pellerin, M. Z. Wei, and Y. J. Zhu (2005), A comparison of the ECMWF, MSC, and NCEP global ensemble prediction systems, Mon. Weather Rev., 133(5), 1076-1097.

Chekroun, M. D., E. Simonnet, and M. Ghil (2011), Stochastic climate dynamics: Random attractors and time-dependent invariant measures, Physica D, 240(21), 1685-1700, doi:10.1016/j.physd.2011.06.005.

Gualdi, S., E. Scoccimarro, and A. Navarra (2008), Changes in tropical cyclone activity due to global warming: Results from a high-resolution coupled general circulation model, J. Clim., 21(20), 5204-5228.

Kuhlbrodt, T., and A. Monahan (2003), Stochastic stability of open-ocean deep convection, J. Phys. Oceanogr., 33(12), 2764-2780, doi:10.1175/ 1520-0485(2003)033<2764:SSOODC $>2.0$.CO;2.

Levitus, S. (1982), Climatological atlas of the world ocean, NOAA Prof. Pap. 13, U.S. Dep. of Commer., Washington, D. C.

Madec, G., P. Delecluse, M. Imbard, and C. Levy (1998), OPA version 8.1 ocean general circulation model reference manual, Tech. Rep. 11, Lab. d'Oceanogr. Dyn. et Climatol., Paris.

Majda, A. J., I. Timofeyev, and E. Vanden Eijnden (2001), A mathematical framework for stochastic climate models, Commun. Pure Appl. Math., 54(8), 891-974, doi:10.1002/cpa.1014.

Palmer, T. N. (2001), A nonlinear dynamical perspective on model error: A proposal for non-local stochastic-dynamic parametrization in weather and climate prediction models, Q. J. R. Meteorol. Soc., 127, 279-304.

Palmer, T. N., and P. D. Williams (2008), Introduction. Stochastic physics and climate modelling, Philos. Trans. R. Soc. A, 366(1875), 2421-2427, doi:10.1098/rsta.2008.0059.

Peixóto, J. P., and A. H. Oort (1984), Physics of climate, Rev. Mod. Phys., $56,365-429$.

Roeckner, E., K. Arpe, L. Bengtsson, M. Christoph, M. Claussen, L. Dümenil, M. Esch, M. Giorgetta, U. Schlese, and U. Schulzweida (1996), The atmospheric general circulation model ECHAM-4: Model description and simulation of present-day climate, Rep. 218, Max Planck Inst. für Meteorol., Hamburg.

Sardeshmukh, P. D., C. Penland, and M. Newman (2003), Drifts induced by multiplicative red noise with application to climate, Europhys. Lett., 63(4), 498-504, doi:10.1209/epl/i2003-00550-y.

Stolle, J., S. Lovejoy, and D. Schertzer (2009), The stochastic multiplicative cascade structure of deterministic numerical models of the atmosphere, Nonlinear Processes Geophys., 16, 607-621, doi:10.5194/npg-16-607-2009.

Stommel, H. (1979), Determination of water mass properties of water pumped down from the Ekman layer to the geostrophic flow below, Proc. Natl. Acad. Sci. U. S. A., 76(7), 3051-3055.

Sun, J., J. F. Howell, S. K. Esbensen, L. Mahrt, C. M. Greb, R. Grossman, and M. A. LeMone (1996), Scale dependence of air-sea fluxes over the western equatorial Pacific, J. Atmos. Sci., 53, 2997-3012. 
Valcke, S., L. Terray, and A. Piacentini (2000), The OASIS coupler user guide version 2.4, Tech. Rep. TR/CMGC/00-10, Cent. Eur. de Rech. et de Form. Av. en Calcul Sci., Toulouse, France.

Wang, X., P. H. Stone, and J. Marotzke (1999), Global thermohaline circulation. Part I: Sensitivity to atmospheric moisture transport, J. Clim., 12, 71-82.

Wilks, D. S. (2008), Effects of stochastic parametrization on conceptual climate models, Philos. Trans. R. Soc. A, 366(1875), 2475-2488, doi:10.1098/rsta.2008.0005.

Williams, P. D. (2005), Modelling climate change: The role of unresolved processes, Philos. Trans. R. Soc. A, 363(1837), 2931-2946, doi:10.1098/ rsta.2005.1676.
Williams, P. D., T. W. N. Haine, and P. L. Read (2004), Stochastic resonance in a nonlinear model of a rotating, stratified shear flow, with a simple stochastic inertia-gravity wave parameterization, Nonlinear Processes Geophys., 11(1), 127-135, doi:10.5194/npg-11-127-2004.

Williams, P. D., E. Guilyardi, R. T. Sutton, J. M. Gregory, and G. Madec (2007), A new feedback on climate change from the hydrological cycle, Geophys. Res. Lett., 34, L08706, doi:10.1029/2007GL029275.

Williams, P. D., E. Guilyardi, G. Madec, S. Gualdi, and E. Scoccimarro (2010), The role of mean ocean salinity in climate, Dyn. Atmos. Oceans, 49(2-3), 108-123, doi:10.1016/j.dynatmoce.2009.02.001.

Williams, R. G., J. C. Marshall, and M. A. Spall (1995), Does Stommel's mixed layer "Demon" work?, J. Phys. Oceanogr., 25(12), 3089-3102, doi:10.1175/1520-0485(1995)025<3089:DSMLW>2.0.CO;2. 